Revue de philosophie et de sciences humaines

e-Portique | 2006

\title{
L'hybridation : un processus décisif dans le champ des arts plastiques
}

\section{Emmanuel Molinet}

\section{(2) OpenEdition}

\section{Journals}

Édition électronique

URL : https://journals.openedition.org/leportique/851

DOI : 10.4000/leportique.851

ISSN : $1777-5280$

\section{Éditeur}

Association "Les Amis du Portique"

Référence électronique

Emmanuel Molinet, «L'hybridation : un processus décisif dans le champ des arts plastiques », Le Portique [En ligne], e-Portique, mis en ligne le 22 décembre 2006, consulté le 10 septembre 2022. URL : http://journals.openedition.org/leportique/851 ; DOI : https://doi.org/10.4000/leportique.851

Ce document a été généré automatiquement le 10 septembre 2022.

Tous droits réservés 


\title{
L'hybridation : un processus décisif dans le champ des arts plastiques
}

\author{
Emmanuel Molinet
}

1 L'hybridation est abordée par cette étude dans le champ des arts plastiques, en privilégiant une analyse du processus et de la notion d'hybride s'y rattachant. L'hybride étant ici non pas une forme symbolique, mais correspondant à la formation d'un objet par l'action d'une multiplicité d'éléments qui, comme l'indique sa définition, crée, génère une nouvelle catégorie de formes, cette dimension dépassant largement celle de l'emprunt, de la combinatoire ou de la superposition pure et simple de techniques ou de pratiques. La figure de l'hybride sera néanmoins interrogée quant à son rôle dans cette histoire complexe, car elle précède la forme. L'histoire de ce processus s'avère délicate en raison de plusieurs points : une terminologie assez récente, un usage courant du terme dans les arts plastiques assez tardif, un manque d'analyse historique approfondie sur ce sujet, ainsi qu'un terme qui en lui-même porte de multiples paradoxes, ambiguïtés, suscitant une multiplicité d'interrogations. L'intérêt de cette analyse étant à la fois de saisir l'évolution de ce terme, au travers de ses différentes acceptions, ainsi que d'observer la complexité de leurs inscriptions et leurs fluctuances, en relation aux différents usages qui participent, modélisent la notion d'hybridation, en caractérisent le processus, dévoilant les différentes strates qui la composent, lui conférant ainsi une identité singulière.

I) L'hybride, forme et identité instable1) Prolifération et généralisation de la forme

2 Si nous assistons aujourd'hui à une prolifération des hybrides, comme l'indique le sociologue Bruno Latour ${ }^{0}$, il apparaît nécessaire de comprendre quelle histoire intègrent ces "objets" et à quelle mutation ils participent ou correspondent. Il semble évident que cette prolifération, si elle se développe non plus dans une sphère ou un domaine, a déjà investi, comme l'écrit Christophe Le Gac, "l'ensemble des sphères productives (médicales, scientifiques, artistiques, voir sexuelles) ${ }^{0}$. Christophe Le Gac précise que ces domaines, en étant confrontés à des problématiques de transformations et d'hybridations, ont finalement subi une mutation décisive, "les identités de la modernité ayant implosé ${ }^{0}$. 
3 Le terme hybride, s'il est devenu un terme courant en étant aussi bien utilisé dans le secteur des nouvelles technologies, de la stratégie, de l'économie, que dans le secteur du management - on parle de forme, de configuration, d'organisation hybride ${ }^{0}$ - agit également comme révélateur d'un monde qui se modélise, se structure à partir d'un modèle de pensée politique. Ce modèle se constitue sur un monde considéré comme monde global, que l'on nomme mondialisation, marquant ici un changement d'échelle décisif à différents niveaux, la mutation étant aujourd'hui planétaire, car essayant d'intégrer à son emprise l'ensemble des sphères, des territoires, des outils dans un même processus de transformation, où cependant en arrière plan se dessine également une volonté d'uniformisation ou d'appropriation qui quelque part mène de manière paradoxale à l'opposé de l'hybridation. Cette mutation généralisée est liée à une autre mutation, celle des "outils", qui voit ses origines et se constitue dans les domaines scientifique et militaire, la science contribuant à la fois à l'apparition du terme dans plusieurs domaines, notamment la biologie et la physique, et à son émergence dans le vocabulaire usuel dans les années 70-80. Un usage récent du terme qui implique, et c'est tout à fait nouveau, l'intervention humaine, une intervention qui nécessairement modifie le cours des choses, transforme les objets ou les éléments y compris parfois par des actes artificiels. Le clonage, la biologie moléculaire, les recherches génétiques, les OGM, l'intelligence artificielle, entrent dans ce processus de transformation où la technologie joue le premier rôle, tout comme dans le domaine militaire. Appréhender les enjeux, les implications et les différents glissements sémantiques du terme hybride, permet de mieux comprendre le processus. L'hybridation n'étant pas un objet en soi, mais l'action de transformer et modifier à la fois les objets, les outils, les pratiques, agissant sur les formes de construction ou réception du réel.

4 Si l'hybridation est actuellement un terme couramment utilisé dans le langage de l'art, il l'est avant tout pour caractériser l'implication des nouvelles technologies et particulièrement des images de synthèse, ainsi que du numérique. Edmond Couchot distingue ce phénomène comme "un nouvel ordre visuel, qui ne définit plus le réel, mais le simule" ${ }^{0}$. Phénomène majeur, puisqu'il met un terme “à un système de figuration perdurant depuis plus de cinq siècles“(...), "l'image numérique substituant au monde réel un monde virtuel" ${ }^{0}$. Un autre aspect de la technologie numérique est qu'elle favorise l'apparition "d'une esthétique de l'hybridation" ${ }^{0}$. Il ne faut cependant pas négliger d'autres usages de ce terme, lesquels tracent d'autres perspectives. Anne Cauquelin ${ }^{0}$ considère, par l'étude de plusieurs caractères significatifs, les installations comme un "modèle hybride". Un ouvrage de référence, Installation, l'art en situation, consacré à cette pratique de l'art contemporain, parle de l'installation comme "discipline hybride" ${ }^{0}$. De nombreux auteurs abordent ce terme depuis peu sous d'autres angles d'approche, tels que Françoise Parfait, Dominique Baqué, Paul Ardenne, Annick Burreau, Florence de Mérédieu... ceci révèle déjà ici une première difficulté, l'hybridation devant être identifiée non plus comme un processus général, mais impliquant au contraire une multiplicité de processus spécifiques.

2) Hybride et hybridation, la construction d'un objet

5 Cette prolifération des hybrides et des formes d'hybridations actuelles implique d'emblée de caractériser quelle en est l'origine et quelles perspectives se dégagent à ce sujet. Une étude de l'histoire de l'art et de l'histoire des œuvres permettra de dessiner l'évolution du processus, en regard de l'apparition des formes, et de l'appréhender 
ainsi au sein d'une archéologie. Celle-ci révèlera les différentes stratifications qui façonnent cet objet. Elle permettra également de comprendre comment les pratiques influencent le domaine de la théorie, et inversement, comment les recherches et études historiques contribuent à faire évoluer ces mêmes pratiques, tout en leur donnant des socles. Ces socles vont contribuer à définir, stabiliser une identité et des zones références dans lesquelles va se constituer ce processus. Ce dernier, en se déterminant dans des zones de références, va développer des caractères spécifiques en fonction des contextes, enjeux et contingences historiques, tout en étant liés à des pratiques, qui par leurs caractères discursifs vont aussi produire, générer ou réalimenter le discours théorique. Ces différentes formes d'échanges favorisent la détermination de cet objet, le rendant lisible dans chaque zone de référence, ce d'autant plus que, comme les pratiques, elles contribuent à faire apparaître des distinctions et paradoxalement aussi des liens entre elles, créant un objet (le processus) portant une multiplicité d'identités. Les usages, les pratiques, les discours et la constitution de l'appareil théorique vont ainsi participer à faire surgir une pratique en tant que champ défini, voire comme à notre époque, en tant que culture, même si ce processus intègre en tant que notion depuis un certain temps déjà une diversité de pratiques, de réflexions et d'approches conceptuelles. Autre intérêt de cette exploration à caractère historique : certains gestes qualifiés de novateurs actuellement pourront au regard d'une analyse de l'archéologie, n'être qu'un prolongement ou le fruit de dialectiques déjà élaborées et dont actuellement nous constatons peut-être une sur-exposition.

Cette recherche imposera de se pencher sur les caractéristiques des œuvres, et de les analyser tant au niveau de leur conception que de leur réception. Les origines du terme hybride, dans les arts plastiques, montrent que la forme et le processus échappent au sens commun, restant porteurs d'ambiguités.

7 De multiples interrogations surgissent, également sur le plan méthodologique, et elles ne sont pas des moindres : un art qualifié "d'hybride" résulte-t-il obligatoirement d'un processus d'hybridation? En quoi l'hybride en tant que figure s'intègre-t-il à la forme, et de qu'elle manière la figure est-elle liée du point de vue artistique, tout comme la forme, au processus?

8 L'histoire nous donne quelques éléments de réponse. En effet, lorsque l'hybride apparaît, c'est en tant qu'être (souvent mi-homme mi-animal), c'est-à-dire figure, et, chose stupéfiante, dès les premières fresques répertoriées et visibles en Namibie (plusieurs milliers d'années avant la grotte Chauvet). Cette figure de l'hybride traverse quasiment la plupart des cultures des peuples du monde. On la retrouve ainsi dans la mythologie grecque ou égyptienne, de même que, en particulier, au niveau des peuples asiatiques et amérindiens. Il faut néanmoins émettre une réserve sur cet hybride, car il n'est pas le résultat d'un processus d'hybridation artistique, étant intégré à un espace symbolique. Il fonctionne comme symbole en désignant par sa signification l'accouplement, le croisement, l'association d'éléments de natures différentes, mais qui sont sur le plan plastique toujours traités dans une même approche stylistique et en cela possèdent une certaine uniformité.

Une évolution se produira lorsque cette figure de l'hybride, progressivement, intégrera des formes de composition de nature hétéroclite, s'inscrivant dans un registre de formes où l'emprunt, la combinatoire, et le mélange, voire l'expérimental, vont impliquer de nouvelles relations entre composition, forme et figure. Ces relations vont générer de nouvelles dialectiques, faisant émerger le processus d'hybridation. Ce que 
l'on nomme art hybride, pourraît apparaitre ainsi comme un art qui provient du croisement de plusieurs formes, engendrant une nouvelle forme, nécessairement extraordinaire. Cette forme extraordinaire, l'hybride, est de surcroît liée au caractère du monstrueux, posant ainsi en prémisse un rapport, une proximité entre la figure et la forme. Ce d'autant plus qu'une corrélation à ce sujet apparaît aussi entre l'hybride et le monstre, lequel justement désigne l'hybride, particulièrement avant l'époque moderne.

10 L'hybride semble inévitablement une forme complexe à caractériser. Deux paradoxes sont à considérer. D'abord l'usage courant de ce terme dans le champ des arts plastiques pouvant être défini comme récent, encore s'agira-t-il ici de dépasser la vision traditionnelle pour saisir les origines réelles de son apparition, une recherche approfondie nous apprenant et ce n'est que le premier paradoxe, que Elie Faure l'a employé dès 1912 dans son ouvrage sur l'histoire de l'art ${ }^{0}$, tandis qu'André Chastel l'a utilisé en $1957^{\circ}$. L'autre paradoxe étant que ces deux historiens de l'art l'ont employé pour des périodes antérieures, Faure à propos de l'époque médiévale et Chastel pour la Renaissance. L'hybride, par cette origine inattendue et méconnue, dévoile différents éclairages qu'il sera nécessaire de resituer pour comprendre une définition de l'évolution de ce processus, la période moderne et la période contemporaine, ainsi que l'art actuel permettant peut être de comprendre les multiples glissements et extensions de ce terme à d'autres significations, usages et enjeux.

11 Dans ces deux cas, c'est le terme hybride qui est utilisé, et il ne l'est pas exactement dans le même sens, même si l'on retrouve plusieurs caractéristiques communes : celle de la forme en général où l'hybride se situe, c'est-à-dire l'ornementation, de même qu'un lien singulier à l'architecture, voire la sculpture ou la fresque. Elie Faure, à ce propos, a justement utilisé cette expression "art hybride" pour qualifier l'art roman. Cet usage n'est pas sans nous rappeler un autre sens que confère à ce terme Victor Hugo, dès 1832, quand, évoquant Notre Dame de Paris, il déclare "cet entassement hybride d'arcades “" $^{0}$, signifiant ainsi la définition courante "composés d'éléments disparates “. Cette définition peut aussi se rapprocher de ce que Chastel distingue en tant que forme hybride, qu'il qualifie "d'ambiguë et monstrueuse“, nous menant vers une autre perspective, le fantastique, directement dans la lignée de la figure hybride. André Breton utilise également ce terme probablement vers 1945, parlant de "cryptogramme hybride", en faisant référence à la peinture d'Ashile Gorky pour "décrire des images oscillant sans cesse entre figuration et abstraction" " ${ }^{0}$, cet artiste inscrivant son œuvre entre le surréalisme et une esthétique abstraite. Ceci permettra de saisir une autre dimension de l'hybridation, qui se joue pendant la période de l'art moderne et annonce certains gestes développés ultérieurement dans l'art contemporain.

3) Complexité et résistance de la forme hybride

13 Cet "art hybride" porte de multiples ambiguïtés et là aussi l'instabilité du terme, sa complexité et ses glissements sémantiques, nécessitent des précautions et imposent de nombreuses interrogations:

14 - Peut-on qualifier d'hybride une forme d'une période antérieure à l'utilisation du terme sans faire un anachronisme?

15 - Comment envisager l'hybride et l'hybridation si ces termes possèdent de multiples sens et nécessitent de les analyser en fonction de lectures distinctes? 
16 - Comment caractériser et distinguer différents processus d'hybridation tout en les resituant dans une évolution ou une chronologie?

17 - De quelles manières les catégories historiques, temporelles et politiques permettraient-elles éventuellement de cerner l'évolution de ce processus sous d'autres angles d'analyse?

18 - L'évolution de l'histoire de l'art et des œuvres, en particulier leur autonomisation, n'impliquent-elles pas un nouveau champ d'action, une mutation à laquelle ce processus participe ou dans laquelle il va pouvoir se déployer?

19 - En quoi la période moderne qui voit une prolifération de gestes novateurs est-elle ou non déterminante par rapport à ce processus?

20 - L'époque contemporaine voyant une prolifération de cette forme, peut-on déceler des facteurs ou éléments qui participent, favorisent ou amorcent cette situation?

21 - En quoi l'art contemporain ne peut-il pas, en tant que nouveau paradigme, déterminer une pensée de l'art nouvelle, dont un des traits significatifs pourrait être de voir l'hybride et l'hybridation comme notion participative à cette pensée?

22 - De nouvelles disciplines comme l'installation ou la performance ne sont-elles pas révélatrices d'une hybridation entre différentes disciplines déjà existantes?

23 - Comment analyser l'effacement ou le brouillage des frontières entre les différentes disciplines artistiques face à ce processus?

4) Précautions méthodologiques

24 Il semble nécessaire, pour comprendre l'apparition et l'évolution de ce processus, de cerner en premier lieu une définition de ces termes - hybride et hybridation -, en analysant les multiples sens qu'ils portent, et les différents usages qu'ils possèdent également dans d'autres domaines artistiques, pour mieux restituer la complexité de leur histoire et les différents contextes dans lesquels ils évoluent.

25 Un autre aspect de cette recherche sera de comprendre dans quelle logique de déplacement l'évolution de ce processus va être impliqué, pour produire, marquer des objets, une histoire, des techniques et des acteurs (artistes, théoriciens de l'art), et comment ce dernier se définit en relation à des contextes, des enjeux, des savoirs, ainsi qu'à sa propre histoire. Observer l'usage de ce terme désormais courant est peut-être aussi révélateur d'une nouvelle acception, d'un changement de paradigme décisif que la période contemporaine a impliqué, imposé.

26 Il faudra également le resituer en fonction de grandes périodes historiques, tout en restituant à cet objet son histoire singulière, pour mieux percevoir les évolutions de la forme et du processus. Pour cela il faudra analyser les œuvres et les différentes catégories de discours qui les accompagnent, de manière à cerner l'implication effective de ce processus, y compris même s'il n'est pas évoqué.

Ce processus devra être compris sous de multiples aspects, ce qui imposera également de ne pas le restreindre au caractère d'un genre ${ }^{0}$ comme le cinéma, le théâtre ou l'opéra, afin d'observer l'émergence concrète de nouveaux contenus, formes artistiques élaborées à partir du croisement de formes antérieures, et qui ne sont donc pas la simple superposition de différentes caractéristiques techniques.

28 Une autre difficulté, dans ces différentes hypothèses, est de saisir précisément quand le processus d'hybridation est mis en jeu et de quelle manière. L'observation des œuvres ainsi que l'appareil théorique historique étant complémentaire et indispensable, pour 
analyser les objets et saisir les différents types d'évolutions que rencontre le processus d'hybridation, ainsi que les multiples champs dans lesquels il se déplace.

II) Penser la modernité : percevoir l'hybridation 1) Le rôle initiateur du romantisme

Un détour par la littérature ou la poésie sera indispensable, l'usage du terme hybride étant, dans ce domaine, antérieur à celui des arts plastiques et, de surcroît, révélateur de multiples aspects parmi lesquels l'usage de nouvelles pratiques propres au romantisme. Un courant majeur qui marque une ligne de partage essentielle entre l'ancien et le moderne, entre des disciplines codifiées et des disciplines amorçant leur autonomie, entre des œuvres dont l'unité et la pureté se désagrège au profit de formes fragmentaires, où l'aléatoire, le montage, tout comme le quotidien, s'imbriquent dans leur élaboration et leur structuration. L'oeuvre porte désormais sa forme comme autonome, car recherchant irréductiblement une indépendance, participant ainsi à un processus d'autonomisation du champ, qui se construit, se constitue progressivement par une transgression des choix et des gestes plastiques.

Cette ligne de partage, qui s'effectue par l'autonomisation des disciplines, nous renvoie au Lacoon de Lessing. Les disciplines se singularisent, tout en autorisant de manière paradoxales l'émergence de liens inédits entre elles. Dans sa dramaturgie de Hambourg (1767 -1768), Lessing envisage l'hybridation ${ }^{0}$ entre le roman et le théâtre, entre la comédie et la tragédie, et donne un sens nouveau au terme hybride : "Que m'importe qu'une pièce d'Euripide soit ni tout récit ni tout drame? Nommez la un être hybride, il suffit que cet hybride me plaise, et m'instruise plus que les productions régulières de vos auteurs corrects tel que Racine et autres... “ ${ }^{0}$ Bannir les genres, chercher de nouveaux sujets ou des formes combinatoires ${ }^{0}$, pour créer de nouvelles formes, le mot d'ordre est lancé, et il éclaire en second lieu les arts plastiques. En effet, cette discipline participera à la révolution engendrée par le romantisme. Chaque art désormais relève d'un champ autonome, ayant les capacités de se construire en fonction de ses propres recherches exploratoires, ce qui comme l'indique Lessing dans cette citation, un des penseurs sur lequel justement le romantisme s'appuie, nécessitera de s'attaquer aux conventions et à l'académisme en vigueur à l'époque pour créer et c'est le paradoxe, de nouveaux objets y compris par l'émergence de nouvelles relations entre différents genres ${ }^{0}$.

31 Par ailleurs, le romantisme, en tant que mouvement, ne se situe pas au sein d'une seule discipline, et c'est une particularité des mouvements d'avant-garde à venir, mais au contraire développe de multiples aspects communs et ramifications dans différentes disciplines telle que la littérature, la poésie, la philosophie, la musique, les arts plastiques... Ce mouvement recherche aussi de nouvelles formes d'associations, et s'intéresse particulièrement au fragment, ces recherches étant liées au concept de totalité organique, induisant le grand œuvre. A cette époque de nouvelles notions comme l'art total, la synthèse, le mélange ou la fusion des arts apparaissent comme majeures, se situant à proximité de l'hybride, tout en étant distinct.

Certains gestes imprévisibles s'opèrent peut-être dans cette volonté d'expérimenter l'œuvre par l'usage des notions de combinatoire ou d'infini, qui elles-mêmes amènent de nouvelles expérimentations touchant à la question de l'hybridation, comme Werner Hoffman le souligne dans la peinture à propos du "retable de Tetschen"(1807-1808). Caspar David Friedrich y élabore une nouvelle forme d'image dans un "lieu intermédiaire entre paysage et image sacrée “" ${ }^{0}$, "le paysage comme icône“, cette expression caractérisant son "rôle hybride “" ${ }^{0}$. Cette question de l'hybridation est 
développée de manière encore plus exemplaire dans le champ de l'écriture, avec notamment Novalis, qui conçoit de nouvelles formes d'écriture.

2) Une histoire à retraverser

Cette réflexion de Werner Hoffman impose de s'interroger sur une question cruciale : un objet peut-il apparaitre alors qu'une terminologie ne s'est pas encore constituée pour l'énoncer, le définir et le délimiter ? On peut procéder comme Michel Foucault par une observation archéologique des savoirs. Cependant, comme il le remarque, "savoir, même dans l'ordre historique, ne signifie pas retrouver et surtout pas nous retrouver (...) c'est que le savoir n'est pas fait pour comprendre, il est fait pour trancher. ${ }^{0}{ }^{0}$ Autre problème, l'histoire d'un même objet peut se présenter en fonction "d'une chronologie (qui n'est) ni régulière, ni homogène ${ }^{\iota^{0}}$, Foucault parlant à ce propos "d'émergence distincte“. Ainsi une pratique peut préexister avant d'être identifiée par un terme spécifique. "Le seuil de positivité est franchi avant celui de son épistémologisation “", comme l'indique Foucault, parlant à ce sujet de "décrochage“.

Interroger le terme hybride, et observer la formation de l'objet, permettra de mieux saisir le processus, pour essayer de fixer son champ d'action. Cela impliquera de le comprendre aussi en relation aux autres termes et notions qui peuvent lui être associées, ou qui se situent à proximité, afin de mieux comprendre son rôle.

Il sera également nécessaire pour cette recherche d'adopter une méthodologie devant prendre en compte l'histoire de l'art et l'histoire des œuvres, signifiant donc l'étude de leurs caractéristiques, y compris en tenant compte de l'évolution récente. Cette dernière permet d'observer le passé autrement, c'est-à-dire en tenant compte de ce que Walter Benjamin avait nommé "image dialectique", et qu'il avait comprise comme essentielle, pour mieux se rapprocher d'une histoire de l'art devant être observée comme un objet évolutif. Une "dialectique à l'arrêt", que Benjamin caractérise ainsi : "ce en quoi l'Autrefois rencontre le Maintenant dans un éclair pour former une constellation".

36 Une histoire qu'il faut "prendre à rebrousse poil ${ }^{\text {“ }^{0}}$, autorisant la prise en compte des "rebuts" ${ }^{0}$. Le rebut étant aussi la forme ignorée, déconsidérée, à laquelle pourtant il faudrait porter attention. Une histoire à reconstituer, celle de la discipline histoire de l'art, telle que Didi Huberman l'appréhende, en s'intéressant au non visible ${ }^{0}$.. L'image de la fresque "madonne des ombres" de Fra Angelico, qu'il analyse dans cet ouvrage, est exemplaire, marginalisée en tant qu'objet à son époque et en tant qu'objet par l'histoire de l'art, alors qu'elle aurait dû susciter de nombreuses interrogations.

Cette occultation est aussi celle d'une pensée de l'art, qui considère l'anachronisme comme faisant partie intégrante de l'histoire, d'une histoire maudite, refoulée, que quelques-uns des grands penseurs comme Carl Einstein, Walter Benjamin, ont néanmoins intégrée à leur pensée de l'art, en formulant un travail théorique "qui peutêtre relu comme une pensée de l'anachronisme“. Une pensée ignorée au point qu'elle n'est quasiment plus relayée ou restituée dans toute sa complexité. L'ouvrage "Devant le temps" de Didi Huberman a le mérite d'approcher ce versant si précieux de l'histoire de l'art, néanmoins porteur d'ambiguïtés, de paradoxes, dont justement sont imprégnées les œuvres et les savoirs.

3) Objet complexe, pensée de la complexité 

hybride, dans sa référence à l'Antiquité, à la Grèce, à l'Egypte, ainsi qu'à de nombreux peuples primitifs, où la mythologie joue un rôle majeur. La figure de l'hybride, cet être monstrueux, incarne en effet en premier lieu un être anormal, car hors du réel. Cette figure va être intégrée à des compositions de plus en plus disparates, prenant un autre visage. Le monstre devient un autre monstre, d'autant plus monstrueux que la composition accentue désormais l'anormalité de l'image. La figure apparaît, elle intègre la forme, la forme impose en elle même le processus, le processus tout comme la figure porte l'anomalie, l'imperfection, l'impureté, la décadence, l'excès. Traits caricaturaux de caractères artistiques, qui de l'époque médiévale à la Renaissance, seront marginaux et marginalisés, souvent incompris, pour être finalement non visibles, ignorés, notamment par les historiens.

péjoratif auquel on assimile ce terme participe également à cette histoire quasiment refoulée. Il nous incite à le comprendre également en fonction de l'étymologie : la formation du mot ibrida en latin nous renvoie à "bâtard", "sang mêlé", amenant ainsi à un acte de transgression qui s'avère inattendu, brisant le cours normal du temps... Ibrida est devenu hybrida par rapprochement avec le grec hubris, signifiant 
"excès, ce qui dépasse la mesure “", voire dans un autre sens, "viol, outrage " “. La démesure comme le viol sont deux éléments perçus négativement, même si les époques moderne et contemporaine attribuent néanmoins une dimension toute autre à la notion de démesure, voire d'outrage.

L'étude sémantique nous révèle également plusieurs sens traduisant ce malaise. Le terme hybridation, dans son sens courant lié aux sciences naturelles, évoque soit "une fécondation qui ne suit pas les lois universelles, soit le fait de croiser deux espèces ou deux genres différents pour provoquer la naissance d'hybrides“ selon l'encyclopédie Universalis.

Cette connotation péjorative est aussi relevée par Etienne Souriau dans son vocabulaire d'esthétique : "on qualifie d'hybrides des œuvres qui mélangent des influences, des styles, ou des genres disparates et mal assimilés, d'où manque d'unité et disharmonie ${ }^{0}$, même si au regard de notre perception actuelle, il semble que cette définition peut être relativisée, voire complétée par d'autres définitions, nous conduisant ainsi à une projection différente de notre regard sur le passé.

5) Une esthétique de la diversité

En effet, malgré ce faisceau d'indices plutôt défavorables, d'autres définitions ou significations plus récentes nous montrent un versant plus actuel et tout autre. L'époque contemporaine, justement, accorde à ce terme une valeur, un sens, qui correspond plutôt à une transdisciplinarité $e^{0}$ de plus en plus favorisée, dans tous les domaines, ainsi qu'à une interaction de plus en plus importante entre les systèmes, les technologies, les savoirs et les cultures, produisant une nouvelle géostratégie. Cette géostratégie se concrétise également avec l'émergence de nouvelles scènes artistiques et de nouveaux enjeux liés davantage à des problématiques induisant une pluralité de contenus.

L'autre point crucial est l'affirmation d'une vision scientifique où l'hybridation est observable comme un processus intégrant diverses mutations dans de nombreux domaines et pratiques : de la biologie au multimédia, de la génétique à la réalité virtuelle, les technologies et les outils aboutissent à une sophistication et à des performances de plus en plus élevées.

$\mathrm{Ne}$ faut-il pas aussi se réapproprier une autre forme originaire de l'hybridation,

peut-être la plus fondamentale et pourtant la plus dissimulée, pour discerner un autre versant inhérent à ce processus, et saisir en quoi il est constitutif, finalement, d'une forme d'expression de la diversité, qui nous renvoie ainsi à l'histoire générale de l'évolution et en particulier du végétal et de l'animal ? Cette perspective nous oblige à regarder la mondialisation sous un autre jour, celui de l'altération des milieux qui coïncide avec l'altération des humanités ${ }^{0}$, c'est à dire des formes d'expressions culturelles, comme du vivant. Il sera donc important d'observer les différences entre hybridation, métissage, et de les comprendre au travers d'autres notions qui interfèrent, comme la mondialisation, la globalisation ou la créolisation, pour analyser les diverses convergences et oppositions qui traversent où distinguent également ces différentes notions.

51 Si l'hybridation est comprise dans le cadre de l'histoire de l'évolution des espèces, elle peut alors être observée comme un processus continu, qui permet à une forme d'être toujours en devenir, c'est à dire de se transformer, se renouveler, et se différencier. 
Partant de ce point de vue, dans le domaine esthétique, elle est donc tout sauf un processus neutre, qu'il faut au contraire envisager comme engendrant des formes de perturbations, voire de ruptures, de transformations incessantes. Ce questionnement nous renvoie à la métamorphose des plantes de Goethe, qui conçoit l'évolution de l'art de manière organique, à l'image de la nature. Une des questions fondamentales est de savoir en quoi elle peut être envisagée, dans le domaine des arts plastiques, comme processus continu. Ou dans quel contexte et dans quelle temporalité elle pourrait s'inscrire pour devenir continue. Questionnement inverse : ne s'inscrit-elle pas dans une temporalité spécifique, en fonction d'un milieu propice?

Une autre interrogation surgit en conséquence : peut-on considérer que ce processus interragit aux côtés d'autres processus, afin de constituer un espace en devenir, un territoire infini, générant lui-même la diversité, et instaurant du même coup un nouveau paradigme de l'art et un nouveau régime des images? Un nouveau paradigme que Rancière distingue par l'émergence d'un régime esthétique de l'art ${ }^{0}$, et que Werner Hoffman analyse comme l'opposition du "mode monofocal" au "mode polyfocal". Il évoque un empirisme monofocal et un "hybridisme polyfocal", tout en ajoutant une nuance, à savoir les nombreuses transitions qui existent entre ces deux pôles. Cette observation mérite d'être approfondie pour plusieurs raisons, particulièrement du fait que l'univers polyfocal peut nous apporter un éclairage intéressant. Cet auteur considère que ce mode n'inclut pas seulement l'art moderne et contemporain, mais intègre justement le médiéval.

Autre interprétation : celle d'André Chastel, pour qui l'art moderne, s'il est un espace exemplaire, l'est encore davantage car il permet de sortir d'une "menace d'étouffement" liée à des catégorisations restrictives et à une forme d'expression limitée, voire autocensurante, pour devenir un espace inédit. Un espace où se propage et peut se déployer une diversité de formes, de pratiques, d'enjeux, de réflexions, sans aucune commune mesure avec le passé. C'est ainsi que se construit une esthétique de la diversité.

Un processus qui néanmoins, semble-t-il, comme dans le domaine des espèces vivantes, et c'est un aspect essentiel, devient opérant lorsqu'il possède les conditions qui lui permettent d'évoluer, et ainsi de s'exercer dans un milieu où il génèrera une diversité. Cette diversité en retour favorise l'hybridation et sa prolifération. Diversité et hybridation sont donc deux constantes intimement liées.

Par ailleurs, comme l'énonce Bernard Blistène, "le projet moderne réfute par sa dimension d'inachevé" (...) "toute forme de clôture “" ${ }^{0}$. Une clôture d'autant plus improbable que les passages, les prolongements, les interactions entre l'art moderne et l'art contemporain sont significatifs, et montrent une pensée de l'art qui se détermine comme pensée de la diversité. Ce qui éclaire la situation actuelle, où l'art contemporain se régénère et s'étend en partie par sa structuration à des scènes extra occidentales, et, par sa capacité à réinterroger des pratiques, une histoire, des données ou des problématiques d'autres champs, formule ainsi de nouveaux projets, voire ouvre de nouvelles issues.

"Réfuter toute forme de clôture", peut aussi dessiner une autre perspective. Ce projet toujours à atteindre, à repenser, n'est-il pas finalement la conséquence la plus exemplaire de cette esthétique de la diversité ${ }^{\circ}$ ? L'art contemporain se retransformant à l'infini, démentant ainsi toute fin de l'art, et offrant des 
horizons toujours possibles, y compris peut-être par des formes d'hybridation de plus en plus subtiles.

6) L'hybride comme forme énonçant la rupture

57 L'hybride, élément révélateur et participatif de cette pensée de la diversité, s'avère dans ce principe - penser la rupture - constitutif d'un nouveau champ, comme une forme irréductible, car elle engendre des formes décisives et dans certains cas annonciatrices de gestes artistiques ou oeuvres ${ }^{0}$ préfigurant de futures disciplines. Une des conséquences s'avère être le caractère inexorable - et autoréflexif- de cette montée en pratique de l'exploratoire, qui permet à l'art de se développer en tant que champ favorisant la critique, la contestation et la transgression.

Pour comprendre la constitution du champ moderne, il faut revenir à une ligne de partage qui se constitue entre l'art ancien et l'art moderne ${ }^{0}$, que l'autonomie des arts formulée par Lessing dans son Lacoon amorçe. L'hybridation peut s'inscrire dans le prolongement de cet événement. Le Lacoon ayant instauré une distinction stricte entre les disciplines, provoquera de manière paradoxale la revendication par les Romantiques de nouveaux rapports entre celles-ci.

Une autre interrogation survient : comment envisager l'hybridation ou l'hybride en relation à la période de l'art moderne, alors que ces termes ne sont pas utilisés dans les manifestes, les écrits ou les textes théoriques pour qualifier des objets et des pratiques, et qu'au contraire d'autres termes sont privilégiés. Autre paradoxe : aujourd'hui de nombreux théoriciens de l'art les utilisent pour déterminer la construction de certains objets de cette époque, en les reliant à des gestes ou des notions comme le collage et le montage, et les nouvelles relations entre poésie, image, son, et action. L'hypothèse la plus plausible serait que l'hybridation, opérant de manière plutôt innatendue, en infiltrant des pratiques, des enjeux, des gestes, des problématiques, demeure quelque part en retrait. Un retrait d'autant plus évident que l'usage courant et les analyses de ce terme s'avèrent très tardifs, notamment dans le domaine des arts plastiques, et qu'il est implicitement lié à l'avènement des arts visuels et à l'hégémonie des nouvelles technologies, en particulier du numérique, dans le prolongement de l'ère du multimédia .

Si l'hybridation participe de ce mouvement irréversible de constitution du champ moderne, elle n'est jamais facilement décelable ou tout à fait évidente, ce qui explique peut-être l'usage peu commun de ce terme à cette époque pour qualifier la transformation ou la mutation des formes, alors que le terme mélange, synthèse, télescopage, syncrétisme, combinaison ou fusion sont eux présents, dans de très nombreux écrits. Une autre difficulté s'amorce ici, celle de ne pas confondre ces notions avec celle de l'hybridation, car elles portent toutes des enjeux et significations différentes, même si dans certains cas précis l'étude de certaines œuvres, en relation au texte leur correspondant pourra révéler qu'un des termes utilisés à l'époque, pouvait impliquer ce que l'on déterminera comme une forme d'hybridation.

61 En effet, ce processus n'intègre t-il pas une mutation conséquente, qu'entraînent plusieurs courants artistiques modernes, en faisant émerger des relations inédites entre l'écrit, l'image, le son, l'acte et l'espace, structurant ces domaines dans un territoire expérimental en devenir, par la génération de nouvelles inventions artistiques ? Ce territoire expérimental n'est-il pas également favorisé par d'autres facteurs : l'évolution et l'invention de nouvelles techniques, qui entraînent de 
nouveaux usages et imposent un autre regard sur le réel et sa captation, à l'image de l'invention de la photographie, qui précipite le devenir de la peinture et participe aussi à son évolution?

62 Un autre aspect majeur, au XIXe et au début du XXe siècle, est l'ouverture des musées ethnographiques et des expositions universelles, qui va susciter un attrait irrésistible des artistes modernes vers les cultures des autres civilisations, favorisant la découverte cruciale des arts orientaux, africains, indiens, et océaniens. Ces influences vont révéler un horizon nouveau, infini, que la plupart des artistes modernes utiliseront pour reconstruire et élaborer un art qui tendra vers des perspectives inédites et novatrices. L'hybridation, dans la constitution de ce champ, va ainsi s'affirmer progressivement, car ce n'est pas un hasard si des théoriciens de l'art dans les arts plastiques, comme Elie Faure et André Breton, ont employé ce terme dans la première période du XXème siècle, ce d'autant plus qu'ils ont approché de manière très approfondie les cultures du monde. Breton de surcroît participera aussi à deux des mouvements artistiques les plus innovants, Dada et le Surréalisme. Ceux-ci développeront justement la logique expérimentale de manière inédite, en inscrivant l'hybride comme un processus mettant en œuvre diverses formes de rupture par différents types de gestes.

III) les usages de l'hybride1) La question du politique

63 Comprendre la question de l'image nécessite aussi de savoir comment un objet, de sa forme d'origine jusqu'à sa forme actuelle, est ou non traversé par la question du politique.

64 Lorsqu' Elie Faure emploie le terme d'art hybride, l'hybridation apparait implicitement pour la première fois en tant que processus, même si elle n'est pas évoquée : c'est-àdire en mêlant des formes de diverses origines issues d'un passé, en particulier celui des guerres de conquête. Des traces, éléments, vestiges qui permettent à un art singulier de se constituer par leur réorganisation, au sein d'une nouvelle forme, l'art roman. Néanmoins, Elie Faure oublie de questionner de manière approfondie les conditions de l'émergences de cette nouvelle forme, qui pourtant s'avèrent fondamentales. Pourquoi cet art émerge t-il de manière hybride ? dans quel contexte ? et en fonction de quelle finalité ? Ces questions se posent également pour la forme et le processus hybride à la Renaissance.

65 Certaines études récentes sont précieuses à ce sujet, car elle dévoilent une hypothèse négligée jusqu'ici : le monstre et le monstrueux désigneraient un ennemi, en particulier le musulman qu'il va falloir combattre. Ainsi en Occident, l'hybride serait en partie instrumentalisé et utilisé en fonction de sa forme symbolique, à savoir le monstre, intégrant ici une politique de l'image. Les images étaient les seuls supports réellement accessibles et compréhensibles, et avaient un impact évident, ce d'autant plus qu'à l'époque, les populations n'avaient pas accès à la lecture de textes.

66 Il faut se rappeler que cette époque correspond à celle des grandes croisades. La religion romaine se prépare à chasser définitivement les Arabes lors des reconquista. Les images auraient donc une force d'incitation à préparer la future croisade. Cette hypothèse est d'autant plus plausible qu'à l'époque, les images sont justement plus facilement comprises que les prêches. L'art roman serait donc en partie un art de propagande. Le commanditaire, l'église Romaine, se sert de la forme et de la figure de l'hybride comme éléments pour accentuer le rejet et le refus de l'identité de l'autre.

67 L'hybride, s'il apparaît ici en tant que forme et processus singulier dans l'histoire de l'art, n'est pas produit dans le champ artistique pour énoncer la rupture, ou dans un énoncé de 
rupture. Tout comme pour la Renaissance, il entre dans un système de représentation, où il correspond à une codification du réel et intègre une politique de l'image, des constructions sociales. Au travers d'une instrumentalisation, il assène un message, participant en cela à une politique de communication. Autre aspect, et il est essentiel, dans le cas de l'art roman comme pour certains grotesques, la figure de l'hybride va s'intégrer à la forme, et en cela montrer une relation figure / forme, forme / composition, qui traduira l'émergence du processus. La notion d'hybride inclut donc différents registres qui qualifient diversement ce que l'on nomme art hybride.

André Chastel nous donne quelques éléments de réponse. L'hybride intègre l'image. Il suggère une nature hostile, "démoniaque“. La Renaissance, en effet, tout en s'édifiant sur la raison, le cartésianisme, la volonté de marquer un territoire de son empreinte, littéralement de le comprendre à son échelle et en fonction de ses lois de perception du réel, accentue le rejet de ce qui est autre. Une empreinte qui porte en fait en elle une indicible violence, liant religion, intérêt économique et politique, d'un Occident qui se prépare à « coloniser » et « soumettre » les autres peuples du monde à ses valeurs, et surtout à ses intérêts. La période antérieure à la Renaissance, l'inquisition, est essentielle à la compréhension de cette capacité d'altération et de dévastation qui intègre la pensée occidentale. Là également, l'hybride sera utilisé en prenant la figure d'êtres extraordinaires, dans des scènes parfois totalement irréelles. Figures incarnant l'être démoniaque, à l'image de la sorcière, notamment pour tout ce qu'elle représente, et par la désignation d'une menace envers l'ordre établi et le dogme.

Concernant l'art roman, comme dans certains grotesques de la Renaissance, on ne peut comprendre l'hybride que sous l'approche d'une politique de l'image. En effet, dans certaines formes hybrides qui apparaissent à ces époques, il semble également qu'une certaine fascination se soit produite pour les productions artistiques de différentes civilisations, ainsi qu'un attrait pour l'expérimentation, qui s'affirme par l'emprunt et par de nouvelles juxtapositions, combinatoires, repoussant les questions de style et de catégorie. Cette expérimentation dans les grotesques est cependant liée à un contexte spécifique, à des interrogations qui émergent aussi dans les champs scientifique et littéraire de l'époque, et qui font apparaître un intérêt pour la "ressemblance et la métamorphose, la variété et l'invention" ${ }^{0}$. L'un des objectif étant justement de constituer de nouvelles grammaires de formes, ceci nous rapproche du principe de la collection et du cabinet de curiosité. L'hybride dévoile une autre perspective. Par son affirmation, il s'inscrit à l'opposé de la peinture d'imitation, touchant ainsi à la question de l'irrationnel ${ }^{0}$.

Dans un cas encore différent, à l'image des êtres qui peuplent les peintures de Gérôme Bosch, une autre vision de l'être hybride s'annonce dans la culture occidentale. Elément singulier, impliquant une forme d'interprétation plutôt positive, il s'avère lié au surnaturel et au fantastique, même si à cette époque ces formes traitant "l'hybride" ont souvent été interprétées comme incompréhensibles, voire inquiétantes.

2) La figure inversée

71 Si la Renaissance, puis l'art académique, consacrent l'imitation du réel, c'est pour mieux astreindre ce réel à un régime de codification. Un art comme forme de communication des valeurs que commandent tour à tour différentes autorités : la royauté, la religion, la noblesse... puis les hauts dignitaires, les révolutionnaires et les élites dirigeantes. Une conception de l'art restrictive, n'autorisant que certains sujets, 
imposant de multiples contraintes, et que progressivement les artistes vont contester, puis anéantir.

La révolution esthétique engendrée par le premier romantisme produit un art dans un espace de l'indéterminé, où tout peut être inventé. Tout reste donc à inventer. Rien ne peut plus désormais contenir ou restreindre une folle et irrésistible ascension vers l'image intacte, vierge, toujours à concevoir, et vers un domaine de l'art compris enfin en tant que domaine auto-réflexif.

Si l'art ancien se sert de l'hybride et de la notion d'hybride, il désigne, par son caractère plutôt hostile, ce qui ne correspond pas au système de valeur accepté. Paradoxalement en ce qui concerne l'art moderne, le processus est perçu au contraire par des critiques comme étant fécond, propice, favorisant l'émergence de formes artistiques novatrices. Cette inversion qui est perceptible récemment dans l'histoire de l'art se conjugue à un autre phénomène, l'entrée dans une culture elle-même devenue hybride, où les télescopages, les interactions, les échanges, amorcés à la fin des années 80 , nous laissent deviner une expansion des processus, des pratiques et des formes.

74 Tout s'invente "dans tout" implique néanmoins un des premiers présupposés de l'art moderne, comprenant les notions d'art total et de chaos infini revendiquées par le romantisme, qui seront reprises dans le projet des avant-gardes. Ce "tout" impliquant que l'art va pouvoir dépasser son propre champ pour investir celui de la vie, et ainsi, entre autres, vouloir transformer les formes et conditions générales d'existence. Un désir d'utopie, qui même s'il s'est avéré peu efficace, a permis de porter de nouvelles idées.

3) L'échec du modernisme

75 Comme le pressent Adorno, les frontières entre les arts se brouillent, mais cette réflexion s'avère partielle, par rapport à une histoire de l'art de plus en plus complexe. Ici d'autant plus, que comme le remarque Rancière ${ }^{0}$, la pensée d'Adorno intègre le Modernisme. Ce courant théorique dont le représentant majeur, Clément Greenberg, incarne une réflexion antinomique avec les notions de mélange, d'hybridation ou d'indistinction, lesquelles sont déconsidérées ou rejetées. Des notions que le Modernisme évacue, instaurant comme principal présupposé que chaque art se joue dans son propre médium et en fonction de ses spécificités. Greenberg invoque un processus "d'autopurification", alors que cette définition, même si elle synthétise un des grands axes de lecture de la modernité, ne peut être que réductrice face à ce que les avant-gardes avaient déjà amorcé. Dès les débuts de la période contemporaine, les œuvres de Jasper Johns, Franck Stella ou Robert Rauschenberg vont irrévocablement rendre caduque cette conception de l'art.

L'importance et le crédit donnés à la thèse de Clément Greenberg marquent davantage l'effondrement d'une pensée de l'art européenne, en exil ou disparue, à laquelle succède une scène de l'art américaine se voulant hégémonique. Celle-ci, tout en intégrant l'héritage européen, s'efforce néanmoins de définir un champ d'action propre. Elle se construit en absorbant divers éléments d'une pensée de l'art européenne, dont cependant elle va nier la complexité réelle, lui restant en partie hermétique.

77 Comme le rappelle Didi-Huberman, il va falloir "repenser dans l'image les rapports de notre maintenant avec l'autrefois“, et restituer ainsi à l'histoire de l'art les pensées complexes de certains historiens non académiques, afin de sortir du modèle positiviste. 
Il sera ainsi possible d'appréhender l'impureté, le chaos, ceci en ne désignant pas une forme négative de l'art, mais plutôt une des aspects fondamentaux permettant de comprendre la structuration de processus et de notions novateurs, ainsi qu'une fonction des œuvres désormais éclatée. Des oeuvres qui possèdent désormais une autre aura, celle de leur intégrité, n'étant plus assujetties à aucun critère immuable, ni définition de l'art, l'absolu devenant la seule quête ou le seul motif de cette recherche.

4) L'hybridation comme processus -notion intégrant l'art contemporain

78 L'art contemporain, en traduisant une nouvelle pensée et définition de l'art, requestionne en profondeur la conception, la définition et l'idée de l'art elle-même. Cette situation entraîne une dématérialisation de l'art, que le processus d'hybridation intègre sur de multiples plans. Un rôle décisif lui est attribué par la participation aux nouveaux enjeux, aux nouvelles déterminations, qui singularisent le champ de l'art contemporain.

79 L'apparition de nouveaux gestes artistiques, qui désormais vont s'affirmer en tant que tels, comme la performance, le happening, l'installation, ainsi que la réinterrogation de pratiques existantes - sculpture, peinture ou photographie - vont impliquer la notion d'hybridation : dans certains cas par une indistinction, une indétermination ${ }^{0}$, un brouillage des catégories ou au contraire un mixage des genres et des pratiques.

Cette redéfinition générale du champ de l'art, désormais, ne se comprend plus dans les questions de représentation et non représentation (abstraction), mais correspond à une problématique des formes intégrant le concept, l'attitude, ou plus généralement la démarche, comme initiateurs d'une œuvre, qui dans certains cas n'est même plus nécessairement réalisée. Cette refondation entraîne à la fois l'apparition de nouvelles pratiques, et l'affirmation d'une relation entre l'art et le langage, l'idée et le processus d'élaboration. Cette dématérialisation implique de nouvelles relations entre art et réel, entre art et contexte, parmi lesquelles l'hybridation devient une notion clé : le processus apparaît dans de multiples cas, parfois de manière visible et décisive, ou parfois par induction ou inclusion, sans être forcément révélé. Elle s'affirme en tant que notion, revendiquée, reconnue, par le rôle décisif qu'elle joue dans les problématiques soulevées par la dématérialisation de l'art.

81 Cette nouvelle forme de pensée de l'art induit une imbrication, une combinaison de gestes multiples qui s'additionnent et constituent l'œuvre. Les oeuvres élaborées prendront aussi bien le statut de performance, de film, de vidéo ou de trace de cette performance, devenant éventuellement une installation, voire restant à l'état de maquette préparatoire. L'hybridation, plus qu'un processus, devient notion, car elle intègre, insère une multiplicité de pratiques, de contenus, qui font, forment de nombreuses œuvres contemporaines.

5) L'hybride comme forme de culture

L'accroissement des dispositifs et installations, omniprésents dans les expositions, a pour conséquence de générer la monstration d'oeuvres de plus en plus hétéroclites, complexes ou mixtes. Ce phénomène s'accompagne de la figure d'un artiste à tendance de plus en plus protéiforme, mobilisant une multiplicité de pratiques et de savoirs au sein d'une même démarche. À cette situation s'ajoute un nouveau contexte: une culture de plus en plus interactive, basée sur la notion d'échanges, de connexions ${ }^{0}$, et de transferts, à laquelle s'ajoutent une culture du remix et de la technologie numérique, qui contribuent dès la fin des années 80 à l'émergence d'une culture 
hybride ${ }^{0}$. Cette culture marque en fait la prolifération d'objets dit hybrides, et l'extension, voire l'apparition de nouvelles formes d'échanges, de conversions ou d'interactions. L'hybridation voit sa présence accrue par l'apparition de nouvelles technologies, qui elles-mêmes favorisent d'autres possibilités d'extension du phénomène à l'image du multimédia, de l'art vidéo et du numérique.

Cette hybridation de plus en plus marquée sollicite de nouveaux enjeux et perspectives, permettant de revitaliser le champ de l'art. L'apparition de nouvelles scènes

extra-occidentales, correspondant au mouvement général de mondialisation qui touche l'ensemble des secteurs, donne la capacité à des artistes de formuler des propositions novatrices, hors de l'éclairage strictement occidental, qui a dominé le vingtième siècle. L'émergence de cette culture hybride doit cependant être comprise dans le prolongement de cette nouvelle pensée de l'art, qui concrétise à la fois les recherches des avant-gardes et de deux des plus grands modèles de formation et d'expérimentation dans le champ de l'interdisciplinarité, que sont le Bauhaus et le Black Mountain College. L'histoire nous révèle que cette culture se déploie et correspond à une esthétique de la contamination ${ }^{0}$, dans laquelle l'hybridation se déploie, devenant aujourd'hui une forme étendue, proliférante.

Si de nombreuses propositions touchent la question du corps, il semble évident que la question de la figure de l'hybride ne nous a jamais quittés. Elle parait amorcer son retour au travers d'un requestionnement du Body art et de l'apparition d'un corps lié de plus en plus à la technologie, nous renvoyant à notre univers, qui lui-même se peuple de plus en plus de prothèses, de greffes, d'une biotechnologie envahissante, jusqu'au clonage, acte ultime d'une hybridation dont les capacités sont de plus en plus étendues et généralisables. Lesquelles peuvent désormais mettre en cause le réel, le corrompre, au sens où il perd sa substance, sa matérialité, et devient réellement fictif. particulièrement dans la période actuelle, il porte également en lui, comme tout autre objet, un autre versant, pouvant engendrer ou posséder des aspects néfastes, voire "monstrueux". Ainsi, s'il est utilisé hors du contrôle de toute forme d'éthique s'il est prétexte à des manipulations artistiques qui ne portent plus d'enjeux, voire ne s'avèrent être que des lieux communs, l'hybridation peut à contrario symboliser l'usure, l'atonie, une certaine forme de repli, que son aspect spectaculaire ne dissimule plus.

\section{NOTES}

0. Bruno Latour, Nous n'avons jamais été modernes, Paris, Ed. La Découverte, 1997, p.7

0. Christophe Le Gac, in Parpaings, Paris, Ed. J. M. Place, 1999.

0. Ibidem 
0. Les Cahiers de l'ERFI ${ }^{\circ} 9$, De l'organisation en hybride aux organisations hybrides : réflexions sur les nouvelles formes d'organisation, Isabelle Coudert-Saderne, Christophe Leyronas, Université de Montpellier I, juin 1996

0. Edmond Couchot, l'art numérique, www.encyclopédia-universalis.fr/ . Voir à ce sujet l'ouvrage du même auteur, l'art numérique, Paris, Ed. Flammarion, 2003.

0. Ibid.

0. Ibid.

0. Anne Cauquelin, Petit traité d'art contemporain, Paris, édition du Seuil, 1996, p156-157.

0. Nicolas de Oliveira, Nicolas Oxley et Michael Petry, Installations, l'art en situation, Paris, Thames \& Hudson, 1997, p7.

0. Elie Faure, Histoire de l'art (1912), Paris, éd. Gallimard, 1981, p 212

0. André Chastel, Le fragmentaire, l'hybride, l'inachevé (1957) in fables, formes, figures, volume

2, Tours, Flammarion, 1978, p. 33 à p.44

0. Victor Hugo, Notre Dame de Paris, Paris, 1832, p 219

0. Une définition du terme hybride au sens figuré que l'on retrouve dans différents dictionnaires comme le Petit Robert

0. Bernard Blistène, une histoire de l'art au XXe siècle, Paris, Beaux arts magazine - hors-série, Centre Pompidou, Déc. 1999, p 103

0. On pourra émettre une réserve sur diverses disciplines qui à l'image de l'opéra ne s'avèrent pas hybrides, mais superposent de multiples techniques ou pratiques, qui coexistent.

0. Muriel Plana, Roman, Théâtre, Cinéma, Adaptations, hybridations et dialogue des arts, Ed. Breal, Clammecy, 2004, p.12

0. G.E. Lessing, Dramaturgie de Hambourg, Didier et cie, 1873, p. 236

0. Voir à ce sujet l'œuvre de Novalis qui sera abordée dans cette étude de manière plus approfondie.

0. Selon Lessing cependant ces différents genres ne s'hybrident qu'au sein d'un même domaine, à l'image de la littérature, qu'il cite.

0. Werner Hofmman, Caspar David Friedrich, Paris, Hazan, 2000, p. 53

0. ibidem, p 53

0. Michel Foucault, "Nietzsche, la généalogie, l'histoire“, in Dits et écrits 1954-1988, II

1970-1975, éd. D. Defert et F. Ewald, Paris, Gallimard, 1994, p. 147-148

0. Michel Foucault, Paris, L'archéologie du savoir, éd. Gallimard, 1969, p. 243-247

0. Ibidem, p. 244 - 247

0. W. Benjamin, Paris, capitale du XIXe siècle, p.478

0. W. Benjamin, Thèses sur la philosophie de l'histoire, 1940.

0. Construire une théorie de l'art à partir d'un montage littéraire nécessite pour Walter Benjamin de considéré tous éléments et surtout le rebus, car il est davantage révélateur de la "préhistoire d'une culture".

0. Georges Didi Huberman, Devant le temps, éd. De Minuit, Paris, 2002, présentation de couverture

0. Ibidem, p.221

0. Ibidem, p40

0. Le Robert Dictionnaire historique de la langue française, Paris, 1992, vol.A-L, p.984

0. Larousse, tome 2, Paris, 1966, p. 518

0. Etienne Souriau, Vocabulaire d'esthétique, Paris, PUF, 1990, p. 840. 
0. Voir à ce sujet l'article de Pierre Berger, Prolégomènes à la conception d'une Université du Futur, Congrès de Locarno, 30 avril - 2 mai 1997 : Annexes au document de synthèse CIRET-UNESCO Centre International de Recherches et Études Transdisciplinaires - http:// perso.club-internet.fr/nicol/ciret/

0. De nombreux peuples ont des conceptions de l'humanité et de leur humanité très différentes.

0. Jacques Rancière, Le partage du sensible, Paris, éd. La fabrique, 2002, p.31

0. Bernard Blistène, Chroniques ironiques de l'art vivant, in Qu'est ce que l'art au xx siècle, Paris, fondation Cartier, 1992, p.42

0. L'expression esthétique de la diversité est utilisée également par Yves Michaud pour caractériser la pensée de Nelson Goodman. Yves Michaud, La crise de l'art contemporain, Paris, Presses Universitaires de France, 1997, p.197.

0. Le Merzbau de Kurt Schwitters, tout comme les actions des Futuristes et des Dadaïstes, annonce respectivement l'installation et la performance comme disciplines. 0. Le mot moderne est utilisé pour désigner les premiers romantiques de l'Athénaeum, l'usage ici n'étant pas habituel, l'histoire pouvant le justifier.

0. Philippe Morrel, "Quelques considérations sur les chimères de la Renaissance", in Cat. Chimères, Arles, 2003, P.34

0. Gilbert Lascaux, le monstre dans l'art occidental, Paris, Ed. Klincksiek, 2004, p.115

0. Rancière, malaise dans l'esthétique, Paris, Ed. Galilée, 2004, p.172

0. Ce terme est à comprendre en relation à de nouvelles réflexions et problématiques énoncées par les artistes, l'intégrité devant être comprise comme une forme d'autonomie que porte l'œuvre en elle même, alors que néanmoins elle déploie en son sein une problématique singulière (qu'elle soit ou non destructrice), liée à une éthique, celle de l'art comme discipline.

0. L'absolu n'est pas ici envisagé comme dans le langage esthétique courant, mais en tant qu'absolu de pensée et d'acte, toujours à atteindre.

0. Ce caractère est déjà utilisé par Gilbert Lascaux, dans son essai de classification des monstres qui justement, sous cet aspect, touche à mon sens à la question de l'hybride, et du composite. Lascaux, 2004, p157à p159.

0. Voir à ce sujet l'ouvrage Connexions, Paris, Ensba, 2002, qui traite des relations entre art et nouvelles technologies.

0. hybridkultur est le titre d'une publication, centrée autour de cette nouvelle forme de culture, parue récemment en Allemagne

0. Paul Ardenne, L'âge contemporain, Ed. du Regard, Paris, 1997, p.297

\section{AUTEUR}

EMMANUEL MOLINET

Doctorant en philosophie à l'université de Metz 